\title{
Narrow Therapeutic Index
}

National Cancer Institute

\section{Source}

National Cancer Institute. Narrow Therapeutic Index. NCI Thesaurus. Code C120270.

A therapeutic index of less than 2-fold difference between the median lethal dose and median effective dose values, or less than 2 -fold difference between the minimum toxic concentration and minimum effective concentrations in the blood. 